\title{
Electrostatic Charge on Pharmaceutical Powders for Pulmonary Application
}

\author{
S. KARNER ${ }^{1}$, N. A. URBANETZ ${ }^{1,2}$ \\ ${ }^{1}$ Institute for Process and Particle Engineering, Graz University of technology, Graz, Austria \\ ${ }^{2}$ Research Center Pharmaceutical Engineering GmbH, Graz, Austria \\ E-mails: stefan.karner@tugraz.at (S. Karner), nora.urbanetz@tugraz.at (N. A. Urbanetz)
}

Sci Pharm. 2010; 78: 623

doi:10.3797/scipharm.cespt.8.PDD34

Pharmaceutical powders intended to target the deeper regions of the lung have to consist of particles with aerodynamic diameters in the range of $0.5 \mu \mathrm{m}-5 \mu \mathrm{m}$. In such fine powders several forces, mainly Van der Waals and electrostatic forces, have strong influence on particle interactions. Particle interactions significantly determine material-loss during powder handling for example in mixing containers, flow-behavior of the powder and the amount of drug released from the inhaler. In addition to this, electrostatic charge affects the deposition of the powder in the lung. Since most pharmaceutical powders are insulators, electrostatic charge is acquired during every contact of the particles among themselves or with other surfaces. The phenomenon of the emergence of electrostatic charge in common powder handling processes has been investigated by several scientists such as Murtomaa [1], Rowley [2] and Zhu [3]. Particularly Zhu examined the charging behavior and influencing variables on the arising of charge of an interactive mixture of salbutamol sulphate and lactose monohydrate in the mixing process.

The aim of this work is to extend these mixing studies with respect to powder blend composition and mixing conditions. The charging behavior is investigated in a T2F Turbula ${ }^{\circledR}$ mixer using a Faraday cup connected to a high resistance electrometer for charge measurement. When a powder is mixed the charge accumulates until a saturated level is reached. The sign and magnitude of the charge and the mixing time required to reach saturation is an important information in the evaluation of the charging behavior of the powder blend. The acquired charge is determined by several factors such as energy of mixing, mixing time, mixing ratio of the blend, relative humidity, particle size, material and wall-roughness of the mixing container. Mixing studies are carried out by systematically altering these factors in order to be able to perform statistical analysis. The results will be highly useful to improve the understanding of the emergence and the impact of electrostatic charge on the performance of pharmaceutical powders especially for pulmonary application.

[1] Murtomaa M, Salvolainen M, Christiansen L, Rantanen J, Laine E, Yliruusi J. Static electrification of powders during spray drying. J Electrostat. 2004; 62: 63-72. doi:10.1016/j.elstat.2004.05.001

[2] Rowley G. Quantifying electrostatic interactions in pharmaceutical solid systems. Int J Pharm. 2001; 227: 47-55. doi:10.1016/S0378-5173(01)00784-0

[3] Zhu K, Tan R B H, Chen F, Ong K H, Heng P W. Influence of particle wall adhesion on particle electrification in mixers. Int J Pharm. 2007; 328: 22-34. doi:10.1016/j.jpharm.2006.07.041 